Revista Latinoamericana de la Papa 19 (1): 59-75

http://www.papaslatinas.org/revista.html

ISSN: 1853-4961

\title{
Estado actual de la producción de papa (Solanum tuberosum L.) en la región andina boliviana
}

\author{
M. Coca - Morante ${ }^{1 / *}$
}

Manuscrito recibido: 06/04/2014

Aceptado: 21/04/2015

Disponible en línea: Junio 2015

\section{Resumen}

El cultivo de la papa (Solanum tuberosum L.) es uno de los más importantes en la región Andina boliviana, tanto por su valor como cultivo de seguridad alimentaria y por su condición de centro de domesticación de una diversidad de papas nativas. Los diferentes procesos sociales, económicos y políticos de los últimos años vienen promoviendo un nuevo panorama de la tendencia de la producción de la papa en Bolivia. La imagen tradicional de país con agricultura eminentemente Andina e Interandina, con la ampliación de la frontera agrícola hacia zonas no tradicionales como los Valles Mesotérmicos, el oriente y chaco boliviano se encuentra configurando una nueva cultura del cultivo y consumo de la papa en Bolivia. El objetivo del presente trabajo fue describir el estado actual de la producción de papa en la región Andina boliviana a partir de la información existente. Se revisaron documentos, informes locales y publicaciones internacionales. Desde 1950, la introducción de tecnología para el mejoramiento de la producción de la papa en los Andes de Bolivia, comenzó apuntando a mejorar la calidad de la semilla de papa a partir de la evaluación y selección de variedades de papas nativas. Después de más de 60 años, en la actualidad, Bolivia, mantiene un sistema de producción y abastecimiento de semilla de papa de calidad certificada. Este sistema desde 1987 a la fecha ha promovido la producción de un número reducido de variedades de papas, entre ellas, la Desirée (Solanum tubersosum subsp. tuberosum) y Waych'a (Solanum tubersosum subsp. andigena). Este esquema junto a los patrones de consumo actual se encuentra modificando la realidad de la producción de la papa en Bolivia, cuyos efectos podrían causar impactos negativos en la conservación de la diversidad de las papas nativas y en la cultura de la papa en la región Andina.

Palabras claves adicionales: Erosión genética, mercado de consumo, cultura Andina, diversidad genética, semilla certificada.

\section{Summary}

The potato (Solanum tuberosum L.) is one of the most important crop in the Bolivian Andean region, as a food security crop value and as a center of domestication of potatoes native varieties. In recent years different social, economic and political processes been promoting a new picture of the trend potatoes production in Bolivia. The andean agriculture, highlands and valleys, country is the traditional image, but with the expansion of the frontier into non-traditional areas such as

Autor para correspondencia: agr.mcm10@gmail.com, m.cocamorante@umss.edu.bo

Departamento de fitotecnia y producción vegetal. Facultad de Ciencias Agrícolas, Pecuarias, y Forestales "Dr. Martin Cárdenas”. Universidad Mayor de San Simón. Cochabamba, Bolivia. 
Mesothermic Valleys, east and Bolivian Chaco is setting a new culture of cultivation and consumption of potatoes in Bolivia. The aim of this study was to describe the current status of potato production in the Bolivian Andean region from existing information. Local reports and international publications were reviewed. Since 1950, the technology introduction to improve potato production in the Bolivian Andean, began pointing to improve the quality of seed potatoes from the evaluation and selection of native potato varieties. After more than 60 years, today, Bolivia, maintains a system of production and supply of seed potato certified quality. This system since 1987 to date has promoted the production of a small number of potatoes varieties, including Desirée (Solanum tubersosum subsp. tuberosum) and Waych'a (Solanum tubersosum subsp. andigena) varieties. This scheme with current consumption patterns are changing the reality of potato production in Bolivia, whose effects could cause negative impacts on the conservation of native potatoes diversity and potato culture in the Andean región.

Additional keywords: Genetic erotion, consuption market, andean culture, genetic diversity, certified seed.

\section{Introducción}

La región Andina boliviana, está conformada, principalmente, por los departamentos de La Paz, Cochabamba, Potosí y Oruro, aunque también, pero, de manera menos significativa, son parte de ella los departamentos de Tarija, Chuquisaca y Santa Cruz, porque su territorio tiene ecosistemas dominantes de Valles (1000$3000 \mathrm{msnm})$ y Chaco (450 msnm), y en el caso de Santa Cruz, con territorio dominantemente amazónico (250 msnm); los otros departamentos de Beni y Pando son plenamente amazónicos (200-250 $\mathrm{msnm})$ y de clima tropical y húmedo.

Los cultivos Andinos, en Bolivia, entre ellos, la papa (Solanum tuberosum L.), se concentran principalmente en los departamentos de La Paz, Cochabamba y Potosí. En el departamento de La Paz, se distribuye la mayor diversidad de papas nativas (cultivadas y silvestres) y sus zonas productoras de papa, se encuentran en el Altiplano (3800 msnm), área circunlacustre del lago Titicaca (3800 msnm) y alturas de montañas húmedas (3000-4500 msnm) influenciadas por la Cordillera Real (Ochoa, 1990); en el departamento de Cochabamba, la mayor diversidad de papas nativas se concentra en las zonas de montaña Altoandinas de Tapacari y montañas
Altoandinas húmedas de Colomi, la producción de papa se concentra principalmente, en las zonas de alturas montañosas (3000-4500 msnm) influenciadas por la humedad de las zonas de transición Andino-Amazónica, en los Valles interandinos (2000-3000 msnm) y zonas de Yungas (1500-2500 msnm), y, en el caso del departamento de Potosí se concentra en pequeñas Altiplanicies del centro y sur de su territorio (3800 msnm) y en las zonas de montaña (3500-4000 msnm) secas, alejadas de los ramales de la cordillera de los Andes (Ochoa, 1990).

En los últimos 60 años, los diferentes procesos sociales, económicos y políticos, por los que ha atravesado Bolivia, han contribuido a configurar un nuevo panorama y una nueva tendencia de la producción agropecuaria de Bolivia. Desde los orígenes de la república y hasta muy avanzada la vida republicana, la agricultura estuvo caracterizada por ser estrictamente de seguridad alimentaria, con su epicentro en la región occidental Andina (Altiplano, Puna y valles Interandinos) (Gandarillas, 2011). Por ello, Bolivia, en el contexto externo, fue reconocida como un país con agricultura Andina, tradicional y de subsistencia. Sin embargo, en el periodo posterior a 1950, esta imagen, en la medida en que fue 
desarrollándose la agricultura en otras regiones no Andinas, como el oriente tropical del departamento de Santa Cruz y posteriormente, la región del Chaco boliviano, sistemáticamente fue modificándose y quedando rezagada hasta alcanzar una nueva imagen (Gandarillas, 2011).

El paso hacia esta nueva situación de imagen de la agricultura tradicional, no significa que fue a costa de dejar la condición de agricultura de subsistencia, sino, que fue para ampliar la condición de país agrícola, de manera que la agricultura tradicional Andina y la agricultura industrial y de exportación, ahora, son eslabones estratégicos para la seguridad alimentaria y la generación de divisas para Bolivia. En el contexto de la imagen de un país con agricultura eminentemente Andina, los cultivos tradicionalmente conocidos como nativos son los tubérculos, entre ellos la Papa (S. tuberosum), y otras raíces Andinas como la oca (Oxalis tuberosa), isaño (Tropaeolum ), etc., los granos Andinos como la quinua (Chenopodium quinoa) y la cañahua (Chenopodium pallidicaule), que son propios y cultivados ancestralmente, y otros cultivos introducidos como el haba (Vicia faba) y Avena (Avena sativa), que también son cultivados desde tiempos de la colonia en la región del Altiplano y Puna alto Andina; los cereales como el trigo (Triticum aestivum), cebada (Hordeum vulgare) y maíz (Zea mays), así como, también las hortalizas como el tomate (Solanum lycopersicum) y la cebolla (Allium sativum), y los frutales de Valle, son cultivados intensivamente en los valles Interandinos (Gandarillas, 2011). Tanto en las alturas como en los valles Interandinos, la producción es parcelaria y en ella se encuentran involucrados miles de pequeños agricultores que practican una tecnología tradicional. El objetivo del presente trabajo fue describir el estado actual de la producción de papa en la región Andina boliviana a partir de la información existente.

\section{La cultura de la papa en la región Andina boliviana}

De entre todos estos cultivos Andinos, la papa (S. tubersoum) y la quinua (Ch. quinoa), por su importancia en la alimentación, regional y mundial, son los que llegaron a identificar a la agricultura boliviana en el contexto mundial (Cárdenas, 1948). Se convirtieron en una especie de iconos imperecederos, cada uno de ellos con su propio valor cultural, económico y político. Varios son los argumentos que sostienen la imagen "Andina" de Bolivia, por ejemplo, su condición de "centro de domesticación de la papa y la quinua" (CIP, 2008) (basado en el cultivo y consumo de una diversidad de papas nativas a lo largo del Altiplano y la Puna alto Andina), su tecnología ancestral (basado en el cultivo en sistemas de conservación del suelo en Terrazas, el sistema de cultivo en Suka kollus en zonas de pampa húmeda, los sistemas de rotación de cultivos basado en las Aynoqhas, el uso de herramientas ancestrales como Ch'akit'aclla, los sistemas de manejo de las predicciones climáticas, etc.), su identidad lingüística (los idiomas nativos como el Aymara y Quechua) y folclórica (la variedad de vestimentas, instrumentos y música nativa), sus valores de reciprocidad y complementariedad, y otros que fueron reconocidos como "saberes Andinos", junto a los cuales fue construida, la "cultura Andina de la papa". En este contexto de mundo Andino, se desarrolló y evolucionó el cultivo de la papa y la seguridad alimentaria, desde tiempos inmemoriales (CIP, 2008).

Con la aplicación de las políticas de modernización para el mejoramiento de este cultivo, se marcó el inicio de un proceso irreversible de quebrantamiento de la cultura 
original de la Papa. Cultura que en el largo plazo estaría condicionada a sucumbir como tal o a subsistir, pero, transformada $y$ adaptada a un nuevo mundo que impone una cultura diferente. En este nuevo contexto, la cultura Andina de la papa, se incorporó, a una especie de ranking mundial, como una de las más atrasadas y a mucha distancia de los países considerados más desarrollados, con el objetivo de reducir la brecha que le separa de otros países, a través del desarrollo tecnológico, social y económico. Los criterios de rendimientos, producción y productividad, comenzarían a ser los referentes que condicionarían su subsistencia de status de agricultura Andina boliviana en el nivel regional y global. Este proceso, en Bolivia, comenzó aproximadamente a partir de los años de 1940. Y uno de los fundamentos de estas políticas fue la introducción de tecnología.

En un estudio realizado el año agrícola 1953-54, se estimaron los rendimientos de una colección de 120 variedades de papas nativas del Altiplano boliviano procedente de recolecciones de semillas utilizadas por los agricultores de la región, y se reportó que el $97,5 \%$ mostraron rendimientos entre 1,0 a 7 t/ha, y la mayor concentración (91 variedades) mostraron entre 1-5 t/ha, así mismo, el autor indicaba que "los rendimiento bajísimos son una consecuencia del ataque de enfermedades degenerativas producidas por virus"2 (Figura 1) (Rodríguez, 1954). De esta manera se infirió que una de las características de la producción de papa en la región Andina fueron los rendimientos bajos atribuidos al uso de semilla de baja calidad. Sobre la base de este argumento fueron elaboradas las estrategias tecnológicas para el

\footnotetext{
2 Desde un punto de vista técnico, los virus, son agentes causantes de degeneración y pérdida de la calidad de la semilla, por tanto, son causantes de pérdidas para el rendimiento y producción.
}

mejoramiento de la producción de papa, es decir, para la obtención de semilla de buena calidad y de nuevos componentes tecnológicos para el cultivo. 
Revista Latinoamericana de la Papa

ISSN: 1853-4961 http://www.papaslatinas.org/revista.html

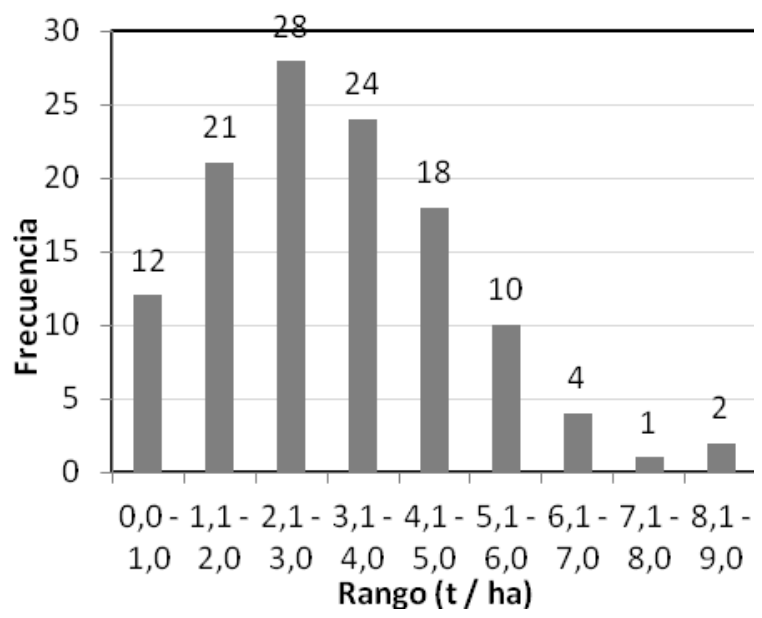

Figura 1. Distribución de frecuencia de rendimiento de 120 variedades de papas nativas (Rodriguez, 1954)

Después de más de 50 años de la aplicación de políticas de mejoramiento tecnológico, se alcanzó a generar una capacidad nacional de producción de semillas de buena calidad. En la actualidad, existe una capacidad instalada (de Instituciones públicas, privadas y mixtas, principalmente de asociaciones de productores) que se encuentran generando anualmente volúmenes considerables de

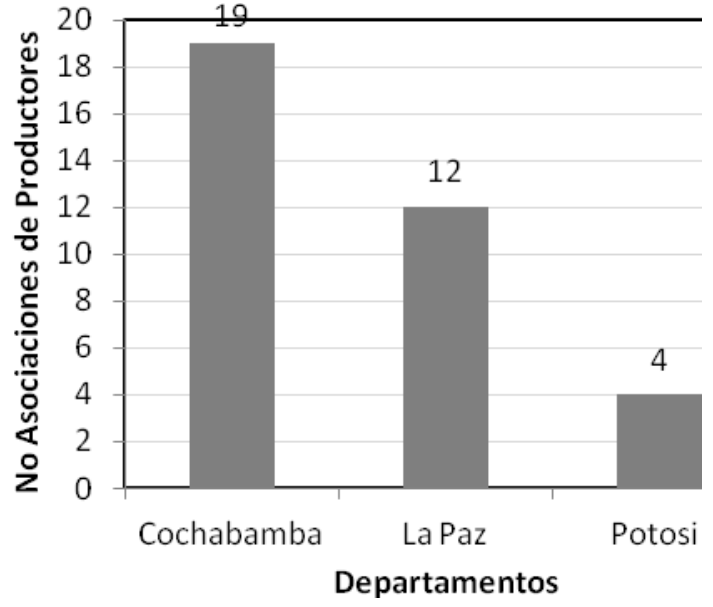

Figura 2. Asociaciones de Productores en tres departamentos de Bolivia (INIAF,2012).

semilla de calidad (fitosanitaria y genética) En el periodo 1987-2005, la producción de semilla de papa en Bolivia, experimentó un impresionante crecimiento de 282 toneladas producidas en 1987 a 6003 toneladas producidas el año 2005 en la región Andina de Bolivia (en los departamentos de Cochabamba, La Paz, Potosí, Chuquisaca y Tarija) (Figura 3) (Torrez y Foronda, 2008). 


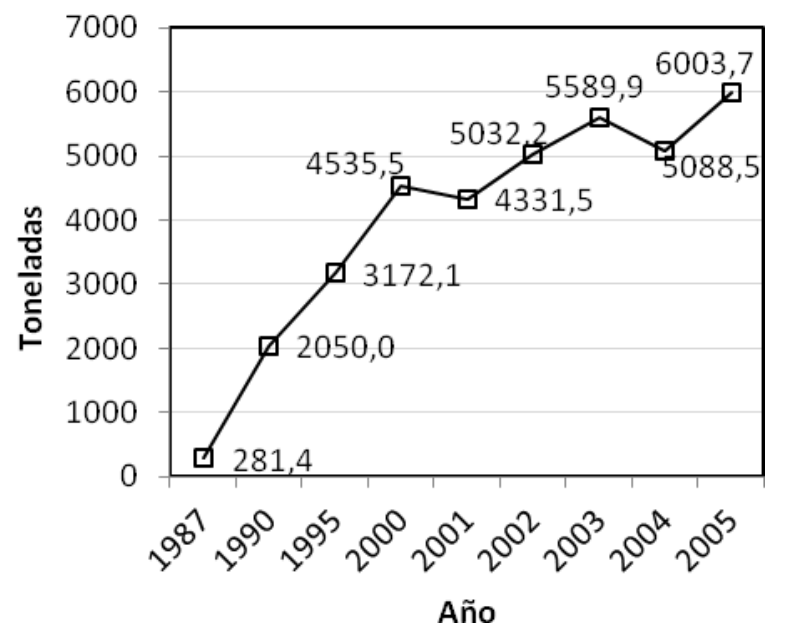

Figura 3. Evolucion de la produccion de semilla de papa en Bolivia (Torrez y Foronda, 2008).

Estos volúmenes producidos en el sistema formal de certificación de semillas, sirvieron para alimentar el sistema de reproducción (o re- multiplicación) de semillas en otras zonas Andinas y no Andinas, así como para alimentar el sistema de la producción tradicional de papa para el consumo en gran parte de la región Andina e Interandina de Bolivia. De manera que, en general, este sistema, en los últimos 20 años, experimentó una renovación de la calidad de semilla que actualmente utiliza $y$ en consecuencia también experimentó un generalizado mejoramiento de la producción.

Por tanto, ya no es posible sostener que los rendimientos sean los mismos o similares (como todavía sostienen las estadísticas del INE (2014) del periodo 1990-2010 donde se observa un similar panorama del año 1949

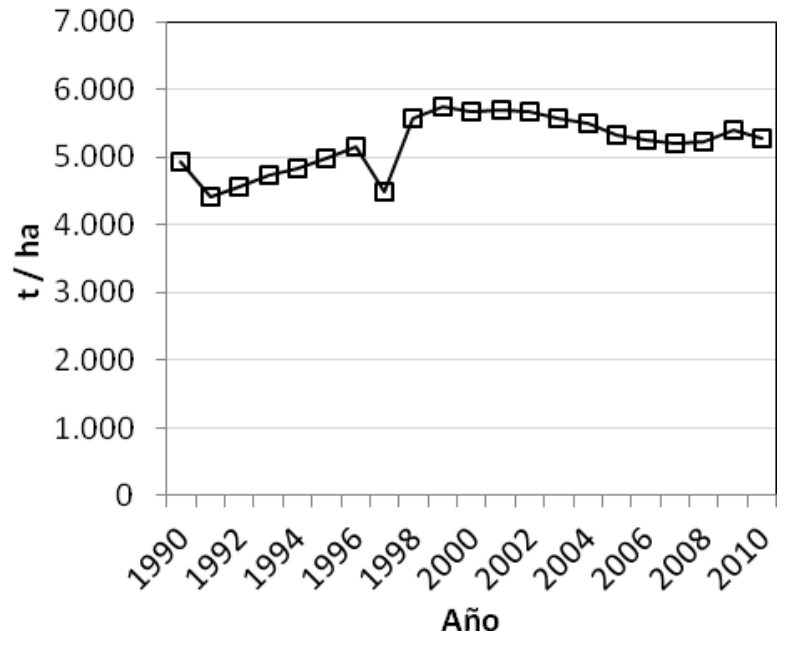

Figura 4. Rendimiento (t/ha) histórico de papa en Bolivia (INE, 2014).

de 4,5-5,5 t/ha. Lo menos esperado es que haya existido una mejora del rendimiento, en unas zonas más que en otras, debido a la heterogeneidad climática y de suelos de la región Andina (Coca Morante et al., 2003). Según, Torrez y Foronda (2008), a pesar de que el uso de semilla certificada en Bolivia alcanza al 3\%, señalan que existe un relativo incremento de los rendimientos a nivel de agricultor $(6,1 \mathrm{t} / \mathrm{ha})$; por otra parte, los diferentes Programas Regionales de Producción de Semilla de Papa, estiman rendimientos promedio a nivel de agricultor entre 8-10 t/ha, y a nivel de las regiones productoras de semilla de papa y regiones usuarias de semillas certificadas, rendimientos entre 8 a 15 t/ha (Coca Morante et al., 2003) (Figura 5A,B). 

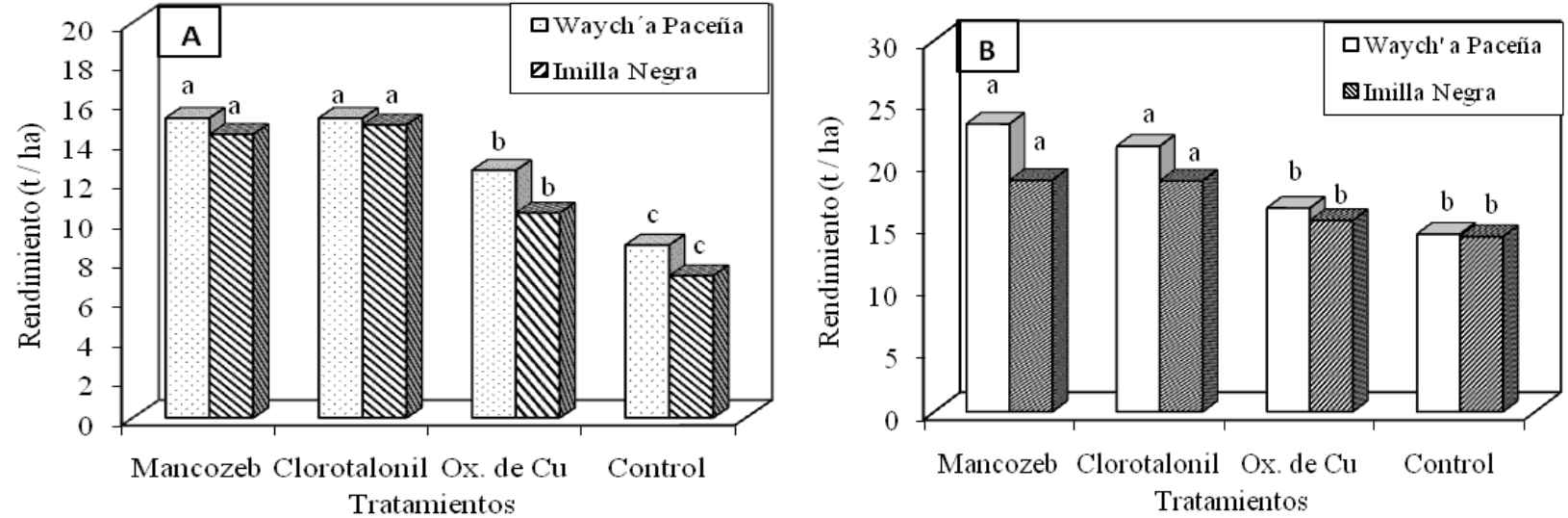

Figura 5. Rendimiento de dos variedades de papas nativas (S. andigena) (Significancia $\mathrm{F}_{0,05}$ ) con diferentes tratamientos de fungicidas en la comunidad de Murumamani, La Paz, Bolivia. A: 1999/2000; B: 2002/03 (Coca-Morante et al., 2003).

Por otra parte, los diferentes factores de orden político, económico y social, también generaron las condiciones para promover un crecimiento de la superficie cultivada de la papa en Bolivia. Hasta 1990, según estadísticas del INE (2014), Bolivia, alcanzó un máximo de superficie cultivada de 140000 hectáreas, pero, con una tendencia que todavía se proyecta para más, porque a partir de este periodo gradualmente se fue generando una época de inflexión, de ser un cultivo eminentemente Altoandino (3000$4500 \mathrm{msnm})$ y del Altiplano (3800 msnm) pasó a convertirse en un cultivo estratégico de las zonas no tradicionales como los valles Interandinos (2000-3000 msnm), valles Mesotérmicos cálidos (1000-2000 msnm), zonas de transición o Yungas (1000-2000 msnm) y el oriente Tropical (250-500 msnm). Las estadísticas del INE (2014) ${ }^{2}$, indican que la superficie cultivada de papa en Bolivia experimentó un crecimiento significativo de 124000 hectáreas cultivadas, en el periodo 2000-2001, a 180000 hectáreas cultivadas en el periodo 2009-2010 (Figura 6). Aunque es difícil aseverar un crecimiento (de hecho ha existido un crecimiento relativo) o afirmar un statu quo de la superficie cultivada en la parte propiamente Andina (porque las zonas tradicionales de ayer son las mismas de ahora), pero, no se estima que sea significativa, por otra parte, se estima que el crecimiento de la superficie cultivada se debe principalmente a la incorporación de las zonas no tradicionales, donde las perspectivas de expansión y crecimiento constituyen un potencial para Bolivia. 


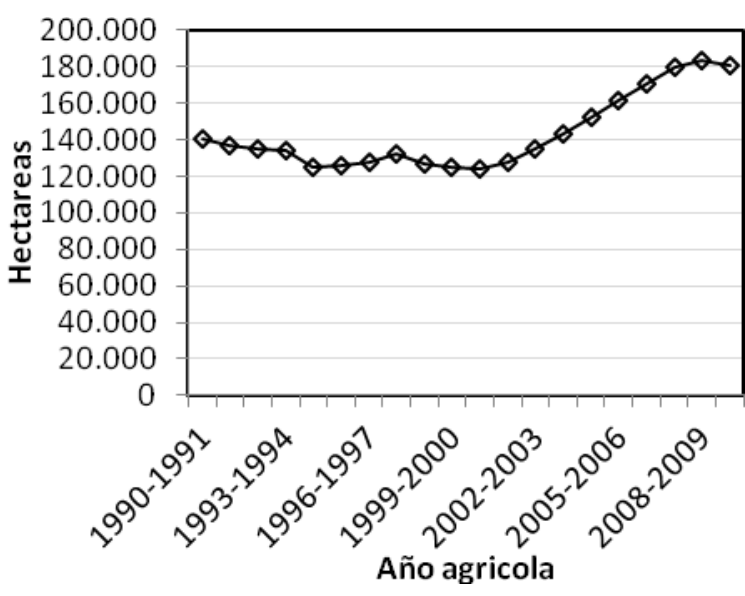

Figura 6. Evolucion de la superficie cultivada de papa en el periodo 1990-2010 (INE, 2014).

Es decir, después de la aplicación de las políticas para el mejoramiento tecnológico, económico y social del cultivo de la papa, se visualiza una nueva realidad en proceso de construcción. En términos de rendimiento, resultaría superflua una comparación entre lo que fue el cultivo de la papa hasta los años 1949, y, lo que es en la actualidad (2014). En este nuevo contexto, el mundo Andino de la cultura de la papa (todavía vigente hasta los años 1940), comenzó a debilitarse a causa de los valores implícitos en la tecnología introducida y a asimilar sus impactos. Existe una nueva realidad notable por los cambios en los patrones de la cultura tradicional. Por ejemplo, el cultivo y consumo ancestral de una diversidad de papas nativas, se encuentra en un impresionante cambio por la preferencia del cultivo y consumo de un reducido número de variedades de papas nativas comerciales (Torrez y Foronda 2008). El año 2005, la producción nacional de semilla de papa fue de 6003 toneladas (Figura 3), pero, con una tendencia a la producción de dos variedades: Désireé (Solanum tuberosum L.) (60\%) y Waych'a (S. andingena) (29\%), y, el restante $11 \%$ para otras variedades y en el orden de importancia siguiente: las introducidas

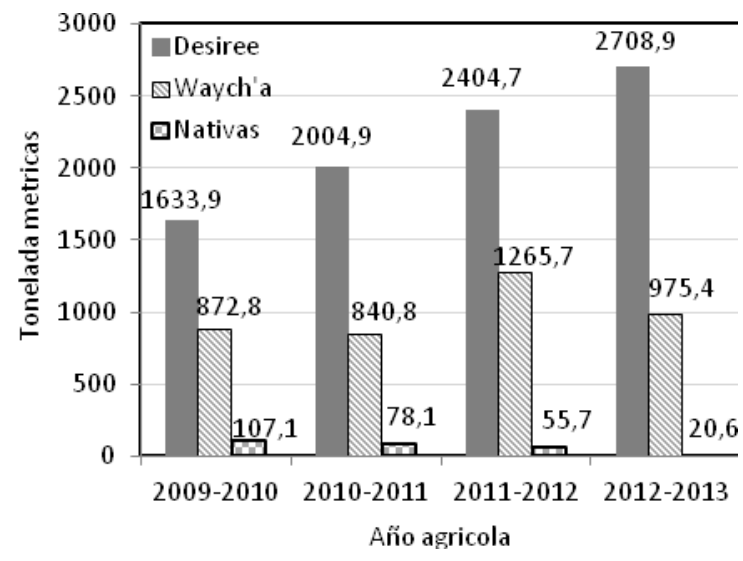

Figura 7. Produccion (TM) de semilla de papa de variedades comerciales y nativas y mejoradas.

(Alpha, Capiro, Monalisa), mejoradas (Musuj, Robusta) y nativas (Imilla Blanca, Imilla Negra, Katawi, Pinta Boca, Sani Imilla y Sinchi Imilla) (Torrez y Foronda, 2008). Desde el año 2010 al 2013, solo el departamento de Cochabamba se produjo una relación de crecimiento inversamente proporcional de las semillas nativas con relación a las variedades comerciales (Figura 7). El año 2009-2010 las variedades comerciales (Désireé y Waych'a) representaron el $95,4 \%$ y las variedades nativas y mejoradas el 4,6\%; el año 20122013 las variedades comerciales representaron el $99.45 \%$ y las variedades nativas y mejoradas $0,55 \%$ (Figura 7) (INIAF, 2012). El año 2010, un año excepcional, en el departamento de Cochabamba, se certificó 27 variedades de papas nativas con una representación del 4,6\% (Figura 7) (INIAF, 2014). El impacto de este cambio resultará drástico para la condición de un país "centro de domesticación de la papa y la quinua" con consecuencias en la cultura tradicional del cultivo de la papa en los Andes bolivianos y la conservación de la diversidad de papas nativas. 
Uno de los ejemplos más patéticos de la pérdida de la diversidad de las papas nativas en la región Andina boliviana, son las papas conocidas como "Papas Imillas" (S. t. subsp. andigena). Según reportes más antiguos sobre el registro de la diversidad de papas Imillas en la región Andina Quechua (Cochabamba y Potosí) y Aymara (principalmente La Paz) (Urquidi, 1984), mencionan a una cantidad aproximada de 41 variedades (aunque es muy probable de la existencia de otra cantidad de papas Imillas distribuidas en los lugares recónditos de los
Andes y no registrados y otras probablemente sean las mismas con la diferencia del idioma) que continuaban siendo cultivadas y consumidas por los habitantes Andinos hasta los años 1960 (Tabla 1). A partir de estas épocas, la historia de las papas Imillas fue sometida a recorrer una ruta en contrasentido. Considerando el centenario o milenario tiempo de la domesticación de la papa, en breve tiempo, esta diversidad de papas Imillas ingresó a un imperceptible camino de pérdida de su diversidad. 
Tabla 1. Variedades de papas Imillas (Solanum tuberosum subsp. andigena) registrada en la región Andina Quechua y Aymara de Bolivia.

\begin{tabular}{|c|c|c|c|}
\hline No. & Especie & Variedad & Fuente \\
\hline 1 & Solanum tuberosum sub sp andigena & Chola Imilla & Luis Felipe Guzmán, 1888 (*) \\
\hline 2 & Solanum tuberosum sub sp andigena & Condor Imilla & Luis Felipe Guzmán, 1888 (*) \\
\hline 3 & Solanum tuberosum sub sp andigena & Imilla Altamachi & Luis Felipe Guzmán, 1888 (*) \\
\hline 4 & Solanum tuberosum sub sp andigena & Imilla Blanca & Luis Felipe Guzmán, 1888 (*) \\
\hline 5 & Solanum tuberosum sub sp andigena & Imilla Pintada & Luis Felipe Guzmán, 1888 (*) \\
\hline 6 & Solanum tuberosum sub sp andigena & Karakara & Luis Felipe Guzmán, 1888 (*) \\
\hline 7 & Solanum tuberosum sub sp andigena & Manzanilla Imilla & Luis Felipe Guzmán, 1888 (*) \\
\hline 8 & Solanum tuberosum sub sp andigena & Negra Pintada Imilla & Luis Felipe Guzmán, 1888 (*) \\
\hline 9 & Solanum tuberosum sub sp andigena & Patrona Imilla & Luis Felipe Guzmán, 1888 (*) \\
\hline 10 & Solanum tuberosum sub sp andigena & Pucañahui Imilla & Luis Felipe Guzmán, 1888 (*) \\
\hline 11 & Solanum tuberosum sub sp andigena & Yana Imilla & Luis Felipe Guzmán, 1888 (*) \\
\hline 12 & Solanum tuberosum sub sp andigena & Zapallo Imilla & Luis Felipe Guzmán, $1888\left(^{*}\right)$ \\
\hline 13 & Solanum tuberosum sub sp andigena & Alco Imilla & Cevallos Tovar, Walter, 1945 \\
\hline 14 & Solanum tuberosum sub sp andigena & Chiar Imilla & Cevallos Tovar, Walter, 1945 \\
\hline 15 & Solanum tuberosum sub sp andigena & Chupic Imilla & Cevallos Tovar, Walter, 1945 \\
\hline 16 & Solanum tuberosum sub sp andigena & Huila Imilla & Cevallos Tovar, Walter, 1945 \\
\hline 17 & Solanum tuberosum sub sp andigena & Imill' Ccoso & Cevallos Tovar, Walter, 1945 \\
\hline 18 & Solanum tuberosum sub sp andigena & Janko Imilla & Cevallos Tovar, Walter, 1945 \\
\hline 19 & Solanum tuberosum sub sp andigena & Kita Imilla & Cevallos Tovar, Walter, 1945 \\
\hline 20 & Solanum tuberosum sub sp andigena & Laram Imilla & Cevallos Tovar, Walter, 1945 \\
\hline 21 & Solanum tuberosum sub sp andigena & Sali Imilla & Cevallos Tovar, Walter, 1945 \\
\hline 22 & Solanum tuberosum sub sp andigena & Alka Imilla & Informe E.E.Belen,1951/52 \\
\hline 23 & Solanum tuberosum sub sp andigena & Anko Imilla & Informe E.E.Belen,1951/52 \\
\hline 24 & Solanum tuberosum sub sp andigena & Panti Imilla & Informe E.E.Belen,1951/52 \\
\hline 25 & Solanum tuberosum sub sp andigena & Allka Chiar Imilla & Informe E.E.Belen,1952/53 \\
\hline 26 & Solanum tuberosum sub sp andigena & Huila Saitu Imilla & Informe E.E.Belen,1952/53 \\
\hline 27 & Solanum tuberosum sub sp andigena & Jacha Chiar Imilla & Informe E.E.Belen,1952/53 \\
\hline 28 & Solanum tuberosum sub sp andigena & Janko Imilla Challi & Informe E.E.Belen,1952/53 \\
\hline 29 & Solanum tuberosum sub sp andigena & Pacco Imilla & Informe E.E.Belen,1952/53 \\
\hline 30 & Solanum tuberosum sub sp andigena & Panti Imilla & Informe E.E.Belen,1952/53 \\
\hline 31 & Solanum tuberosum sub sp andigena & Chuchi panti Imilla & Informe E.E.Belen,1953/54 \\
\hline 32 & Solanum tuberosum sub sp andigena & Janki Imilla & Informe E.E.Belen,1953/54 \\
\hline 33 & Solanum tuberosum sub sp andigena & Sako Imilla & Informe E.E.Belen,1953/54 \\
\hline 34 & Solanum tuberosum sub sp andigena & Imilla Rosada & Cárdenas, 1963 \\
\hline 35 & Solanum tuberosum sub sp andigena & Lumku Imilla & Cárdenas, 1963 \\
\hline 36 & Solanum tuberosum sub sp andigena & Puca Imilla & Cárdenas, 1963 \\
\hline 37 & Solanum tuberosum sub sp andigena & Sakoñahui Imilla & Cárdenas, 1963 \\
\hline 38 & Solanum tuberosum sub sp andigena & Sani Imilla & Cárdenas, 1963 \\
\hline 39 & Solanum tuberosum sub sp andigena & Taka Imilla & Cárdenas, 1963 \\
\hline 40 & Solanum tuberosum sub sp andigena & Janko Chockella & Ochoa, 1990 \\
\hline 41 & Solanum tuberosum sub sp andigena & Wila Monda & Ochoa, 1990 \\
\hline
\end{tabular}

(*) Citado por Urquidi (1984) 
En la actualidad, de las 41 variedades mencionadas, se cultivan una reducida variedad de las papas Imillas en la región Andina tradicional, por ejemplo, la Imilla Negra o Yana Imilla (Quechua) o Ch'iar Imilla (Aymara), Imilla Blanca o Yurac Imilla (Quechua) o Janko Imilla (Aymara), Wila Imilla (Aymara) o Puca Imilla (Quechua), Sani Imilla y probablemente alguna que otra papa Imilla más. De esta cantidad de papas Imillas llegan a los mercados de consumo local y de las ciudades, una cantidad aún más reducida, por ejemplo, la Imilla Blanca o Yurac Imilla (Quechua) o Janko Imilla (Aymara), en menor proporción la Imilla Negra o Yana Imilla (Quechua) o Ch'iar Imilla (Aymara) y en mucho menos cantidad por ejemplo la papa Sani Imilla o la Wila Imilla. Es decir, un impacto drástico a la condición de país "centro de domesticación de la papa".

\section{La emergencia de una nueva cultura de la papa}

La agricultura Andina, también caracterizada como de subsistencia, satisface principalmente el autoconsumo y temporalmente a los mercados locales (rurales y urbanos) del occidente boliviano. Este sistema de producción, en el pasado y en la actualidad, ha mostrado sus propias debilidades, con consecuencias de desabastecimiento crónico en los mercados de alimentos. Aparentemente resulta contradictorio hablar de desabastecimiento de papa en los mercados, cuando por lo expuesto se considera que la agricultura Andina habría alcanzado un mejoramiento cualitativo y cuantitativo en la producción de papa, pero, es una realidad que tiene historia la introducción de papas desde otros países a Bolivia para satisfacer la demanda en los mercados urbanos (Gandarillas, 2011). En dependencia de la perspectiva de análisis del problema, este tiene una serie de aristas que complejizan la misma, pero, por una parte, y en ciertas épocas, el abastecimiento de papa de otros países se convierte en una necesidad debido a los elevados precios internos, y por otra parte, en otras épocas, debido a la baja producción nacional por causas de orden económico, político o de origen natural como las sequias, heladas, granizadas o inundaciones.

Aunque las heladas y sequias históricamente fueron los fenómenos naturales más drásticos para la agricultura Andina. Se conocen de varios ejemplos históricos de sequias, como la sequía en los años de la guerra del pacifico (1879) con efectos dramáticos en el abastecimiento de productos agrícolas en general, o la sequía nacional de los años 1983-84, que ocasionó desabastecimiento de tubérculos, cereales como maíz y trigo y hortalizas, etc.; en el caso de las heladas, conocido como un fenómeno recurrente en las región del Altiplano y zonas Altoandinas en general, que históricamente también causaron pérdidas en la producción y desabastecimiento de papa; y, las abundantes lluvias que en las regiones Andinas húmedas que ocasionan pérdidas debido a la severidad del ataque de plagas y enfermedades (que causan pudriciones como la khanura $^{3}$, khasahui ${ }^{4}$, etc.), e inundaciones en los valles Interandinos (Gandarillas, 2011). Estos fenómenos no fueron aislados, sino, recurrentes, a lo largo de la historia de la agricultura Andina, aunque todos con diferente grados de intensidad de daño, pero, con efectos negativos en la seguridad alimentaria.

En general, estas causales, han promovido el desarrollo de mecanismos de respuesta a partir de la sociedad y del Estado. Por una parte, la sociedad, generando sus propios

\footnotetext{
${ }^{3}$ Palabra de origen Quechua que significa pudrición del tubérculo.

${ }^{4}$ Palabra de origen Aymara que significa follaje quemado.
} 
mecanismos de subsistencia, y por otra parte, el Estado, generando políticas en un marco más global y de largo plazo. Pero, desde ambas direcciones, buscando el abastecimiento de alimentos, y por lo tanto, paleando la fragilidad del sistema con la creación de un mercado de alimentos para los productos de otros países, principalmente, vecinos, como por ejemplo, la crónica importación de papa, desde la Argentina o el Perú, la importación de harina y otros cereales, en el pasado inmediato de los Estados Unidos y en la actualidad de la Argentina, la importación de frutas y hortalizas durante gran parte del año desde la Argentina, Chile y Perú, etc. (INE 2014) Es decir, el sistema Andino de reproducción de la seguridad alimentaria es todavía incuestionablemente frágil.

Con el transcurrir del tiempo, la expansión del cultivo de la papa cobró un sentido de estrategia nacional, necesaria, complementaria y sostenible en el largo plazo. Porque la producción tradicional demostró capacidad de sostenimiento temporal del mercado principalmente occidental (es decir, por unos meses del año) y complementada con la producción de las siembras de tardías $\left(\right.$ Lojru $^{5}$ ) y de invierno

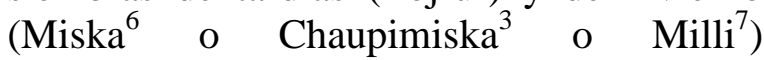
practicadas en los Valles interandinos, y por otra parte, porque no alcanzan a satisfacer los mercados emergentes de Bolivia, como es el caso del oriente boliviano, tanto en volúmenes como en precios y calidad (Torrez y Foranda, 2008). Una de las características del mercado oriental boliviano, o emergente, es la dimensión de

\footnotetext{
${ }^{5}$ Palabra de origen Quechua, utilizado principalmente en Cochabamba.

${ }^{6}$ Palabra de origen Quechua, utilizado en toda la región Andina, principalmente, Cochabamba, Chuquisaca y Potosí.

${ }^{7}$ Palabra de origen Aymara, utilizado en la región Andina, principalmente, La Paz.
}

su mercado, es decir, es uno de los mercados más grandes de consumo de Bolivia debido al crecimiento poblacional de los últimos 50 años, de ser el quinto departamento en población el año 1950 (244.658 habitantes) al año 2012 paso a ser el segundo departamento más poblado de Bolivia (2.655.084 habitantes) a muy poca diferencia de La Paz (2.706.351 habitantes) (Figura 8) (INE, 2014). 


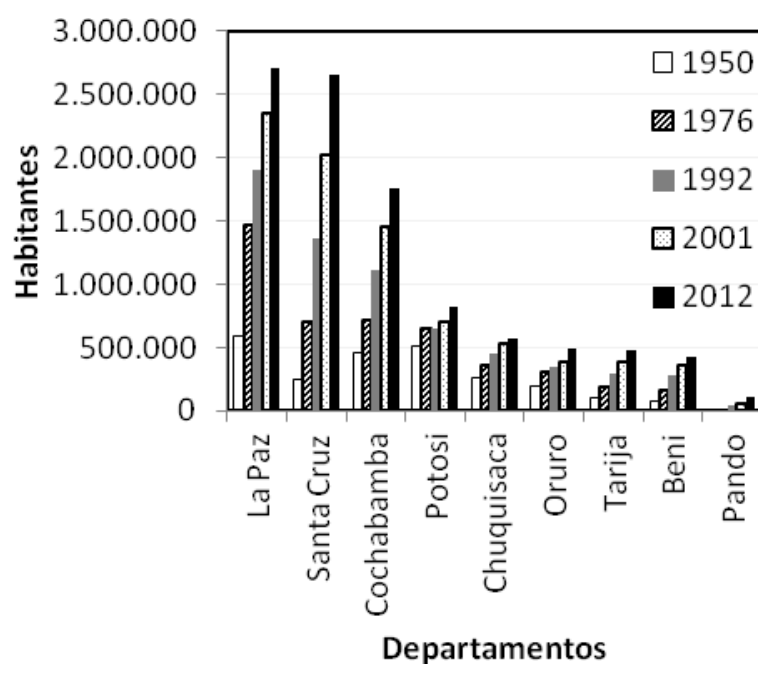

Figura 8. Crecimiento de la población (habitantes) en Bolivia: 1950-2012 (INE, 2014).

La expansión del cultivo de la papa en zonas no tradicionales, también generó una nueva tendencia cultural, social, económica y tecnológica. Al parecer, nos encontramos en las postrimerías de una cultura tradicional Andina y en la configuración de una "nueva cultura" de la papa a partir de nuevos contextos agroecológicos y socioeconómicos, como el Oriente boliviano. Esta nueva cultura se fundamenta en paradigmas también diferentes a los Andinos, como son el "mercado y los precios" (INE, 2014).

En esta perspectiva, la demanda de los nuevos mercados emergentes impone, incluso, arrastran a los mercados tradicionales (occidentales) hacia nuevos hábitos de consumo que con el tiempo podrían ser los nuevos patrones de consumo. Estos hábitos seleccionan y terminan imponiendo determinados tipos o clases de papas que deben cultivarse (en ambos sistemas agroecológicos: Andino tradicional y no Andino), orientando la perspectiva de la demanda por un número preferente de variedades de papas que reúnan requisitos, como por ejemplo, que sean, en suma, fáciles de pelar y adecuadas para las comidas rápidas. Estas preferencias describen bien a las variedades introducidas antes que a las variedades nativas, con excepción de algunas, la gran mayoría poseen características diferentes a las requeridas.

Por otra parte, si los hábitos de esta demanda, generan un efecto de impacto en la cultura tradicional de la papa donde ancestralmente se cultivaron una diversidad de papas nativas, en el largo plazo podrían terminar siendo desplazadas por pocas variedades introducidas para los mercados emergentes y por otras pocas papas nativas (Waych'a) para los mercados occidentales tradicionales y para el autoconsumo. Aspecto que se denota a partir de la orientación que tiene la producción alto Andina de semilla de papa apuntalando al mercado oriental. Según, datos del año 2005, de las 6003 toneladas de semilla de papa producidas en las zonas tradicionales alto Andinas de Cochabamba y Potosí (Figura 3), aproximadamente el $60 \%$ de esta producción representa la variedad Désireé y el $80 \%$ de esta producción se comercializa en los Valle Mesotérmicos (Torrez y Foronda, 2008). Al presente, debido al cultivo extensivo de la 
papa en el oriente boliviano del departamento de Santa Cruz (250 msnm), en especial con la variedad Désireé, se estima que los volúmenes de semilla certificada producidos en el sistema Andino resultan insuficientes, razón porque esta demanda estaría generando, incluso, el uso de semilla procedente del sistema informal (a partir de las ferias alto Andinas, principalmente de Cochabamba y Potosí).

El crecimiento de esta actividad semillera en las zonas alto Andinas, se sostiene, principalmente, por la oportunidad de los precios que resultan favorables para los productores y por la calidad de su semilla. Los precios, permanentemente, fueron favorables a los productores debido a la existencia de un amplio mercado nacional, a la gradual toma de conciencia de parte de la gran mayoría de los productores Andinos y no Andinos sobre las cualidades del uso de semilla certificada y al sostenido apoyo institucional (cooperación internacional). Y por otra parte, la calidad de la semilla producida en las zonas de alturas Andinas, es muy valorada por los productores en general, debido a los buenos a excelentes rendimientos que obtienen cuando utilizan semillas producidas en estas alturas y mucho más cuando son semillas certificadas (Torrez y Foronda, 2008; Gandarillas, 2011). Este sistema que deriva del antiguo sistema de reciprocidad y complementariedad Andina, se ha convertido en la actualidad como el único sistema en Bolivia (Torrez y Foronda
2008), aunque probablemente sea generalizado en otros países de la región Andina como Perú, Ecuador, etc. El potencial de rendimiento de esta semilla se encuentra relacionado con la cantidad de horas frio a la que se encuentra expuesta la fisiología de la planta para la formación de tubérculos y a las bajas poblaciones de insectos (pulgones y trips) vectores de virus causantes de la degeneración de la semilla que tiene que ver con la sanidad. Es decir, el sistema funciona produciendo semilla en las alturas alto Andinas (3000-4500 msnm) para utilizar en la producción de papa consumo en los Valles y Llanuras orientales (250$3000 \mathrm{msnm}$ ) hasta dos años consecutivos y la semilla pierde su capacidad productiva (conocida por los agricultores como "semilla cansada"), y entonces, nuevamente a las alturas y continúa el ciclo.

Las zonas alto Andinas productoras de semilla de papa (donde también alcanzaron un alto grado de organización de los productores como las Asociaciones de Productores) (Tabla 2), en general, experimentaron un impacto económico y social muy importante en el conjunto de la comunidad campesina. Al presente, debido a esta actividad y a su significación económica, muchas zonas alcanzaron a consolidarse como centros especializados en la producción de semilla de las variedades más demandadas por los mercados con efectos de impacto importantes en la realidad social y económica de las familias rurales. 
Tabla 2. Asociaciones de Productores de Papa de diferentes departamentos de Bolivia. (Fuente: INIAF, 2014; Embajada de Bolivia en China, 2014.).

\begin{tabular}{|c|c|c|c|}
\hline Nombre Asociación & Acrónimo & Departamento & Municipio \\
\hline Asociación de Productores de Tapacari & APROASAM & Cochabamba & Tapacari \\
\hline Asociación de Productores de Sacaba & APROSECAP & Cochabamba & Sacaba \\
\hline Asociación de Productores de Semillas & APROSEMILLAS & Cochabamba & Cocapata \\
\hline Asociación de Productores de Semilla de Papa & APROSEPA & Cochabamba & Cocapata \\
\hline Asociación de Productores de Tikipaya & APROSER & Cochabamba & Tikipaya \\
\hline Asociación de Productores de Papa & ASEP & Cochabamba & Cocapata \\
\hline Asociación de Productores de Papa & ASEPA & Cochabamba & Cocapata \\
\hline Asociación de Productores de Papa & ASOFLOR & Cochabamba & Cocapata \\
\hline Asociación de Productores de Papa & ASPAN & Cochabamba & Cocapata \\
\hline Asociación de Productores de Papa & ASPCHA & Cochabamba & Cocapata \\
\hline Asociación de Productores Mosoj Tarpuy & MOSOJ TARPUY & Cochabamba & Cocapata \\
\hline Asociación de Productores de Papa & ORPACA & Cochabamba & Cocapata \\
\hline Asociación de Productores Sumaj Poqoy & SUMAJ POQOY & Cochabamba & Cocapata \\
\hline Asociación de Productores Sumaj Muju & SUMAJ MUJU & Cochabamba & Cocapata \\
\hline Asociación de Productores Sumaj Tarpuy & SUMAJ TARPUY & Cochabamba & Cocapata \\
\hline Asociación de Productores Tunari & TUNARI & Cochabamba & Quillacollo \\
\hline Asociación de Productores Totora & ARAPI & Cochabamba & Totora \\
\hline Asociación de Productores Pocona & ARAPI & Cochabamba & Pocona \\
\hline Unidad Producción de Semilla-Semilla de Papa & UPS-SEPA $(*)$ & Cochabamba & Pocona \\
\hline Unidad Producción de Semilla-Semilla de Papa & UPS-SEPA $(*)$ & Cochabamba & Totora \\
\hline Unidad Producción de Semilla-Semilla de Papa & UPS-SEPA $(*)$ & Cochabamba & Cocapata \\
\hline Asociación de Productores de Murumamani & ACSEM & La Paz & Achacachi \\
\hline Asociación de Productores de Murumamani & APASOM & $\mathrm{LaPaz}$ & Achacachi \\
\hline Asociación de Productores de Combaya & APROSC & $\mathrm{LaPaz}$ & Larecaja \\
\hline Asociación de Productores de Sica Sica & APROSEPT & $\mathrm{LaPaz}$ & Aroma \\
\hline Asociación de Productores de Murumamani & ASEMM & $\mathrm{LaPaz}$ & Achacachi \\
\hline Asociación de Productores de Murumamani & ASEPROMAPRIA & $\mathrm{LaPaz}$ & Achacachi \\
\hline Asociación de Productores deChococopa Grande & ASISCHOG & $\mathrm{LaPaz}$ & Achacachi \\
\hline Asociación de Productores de Pintucuni & CHAMANI JATHA & $\mathrm{LaPaz}$ & Colquiri \\
\hline Asociación de Productores de Chococopa Chico, & $\begin{array}{l}\text { PROSAN } \\
\text { SEMILLEROS }\end{array}$ & $\mathrm{LaPaz}$ & Achacachi \\
\hline Asociación de Productores de Chiaraque & CHIARAQUE & La Paz & Yaco \\
\hline Asociación de Productores de Mecaña & COPROSEM MECAÑA & $\mathrm{LaPaz}$ & Yaco \\
\hline Asociación de Productores de Cupunuta & PROY.FAO-Sem. Andinas & $\mathrm{LaPaz}$ & Patacamaya \\
\hline Asoc. De Prod. De Semilla de papa Villalón & APROSEPA & Potosí & Villazón \\
\hline Asoc. de Prod. Semilleristas de papa-Betanzos & ASEPA & Potosí & Betanzos \\
\hline Asoc. de Peq. Prod. Sem Papa Ayllu Layme Pukara & APPA-LM & Potosí & Uncía \\
\hline Agropecuaria Solidaria & AGROSOL & Potosí & Villazón \\
\hline
\end{tabular}

(*) Empresa mixta Productores-Estado. 


\section{ARTÍCULO DE INVESTIGACIÓN}

Revista Latinoamericana de la Papa http://www.papaslatinas.org/revista.html En conclusión, se encuentra notablemente visible la emergencia de una nueva realidad de la producción de la papa en Bolivia. Los paradigmas Andinos de la cultura tradicional de la papa se mantuvieron intactos hasta la introducción e implementación de políticas para el mejoramiento tecnológico de este cultivo. A partir de la cual, y luego de un proceso prolongado, se viene configurado un sistema Andino de producción de semilla con impactos económicos y sociales en la realidad de la vida rural e institucional y que se encuentra interactuando con nuevas realidades como el mercado y los precios, los que su vez vienen incidiendo en la orientación de la emergencia de una nueva cultura de la papa en Bolivia.

\section{Conflictos de interés}

El autor expresa que no hay conflictos de interés con la publicación de este artículo.

\section{Agradecimientos}

El autor desea expresar su agradecimiento al Ing. Yuri Antezana, técnico del Instituto Nacional de Innovación Agropecuaria y Forestal (INIAF, Cochabamba), por su colaboración en la proporción de datos estadísticos de producción de semilla regional. A la Ing. M.Sc. Dora Ponce Camacho, Docente de la FCAyP - UMSS e Investigadora del Centro Universitario Agroecología Universidad Cochabamba (AGRUCO), por su comentarios y sugerencias al presente documento.

\section{Referencias citadas}

Cárdenas M. 1948. Plantas alimenticias nativas de los Andes de Bolivia. Folia Universitaria,, Bolivia II (2): 36-51.

Cárdenas M. 1963. Germoplasma de papas cultivadas acumulado en Bolivia durante los últimos seis años. Folia Universitaria, Bolivia. IX: 58-87.
ISSN: 1853-4961

Coca-Morante M., W. Castillo-Plata y Álvaro Méndez. 2004. Control quimico de la Septoriosis (Septoria lycopersici Speg.) de la papa (Solanum tuberosum subsp. andigena) en zonas andinas de altura de Bolivia. Revista Latinoamericana de la Papa, Perú. Vol 18 (1): 107-121.

Centro Internacional de la Papa (CIP). 2008. The Odyssey of the Potato - La Odisea de la Papa. Centro Internacional de la PapaMinisterio de Agricultura y Relaciones Exteriores del Perú. Centro Internacional de la Papa. Lima, Perú. 45 p.

Estación Experimental Toralapa. 2014. Banco de Germoplasma de los Recursos Genéticos de la Agrobiodiversidad. Ministerio de Desarrollo Rural y Tierras, Instituto Nacional de Innovación Agropecuaria y Forestal (INIAF). Cochabamba, Bolivia. Bípticos. 4 p.

Embajada de Bolivia en China. 2014. Lista básica de OECAS en Bolivia. http://www.embolchina.com/businessXs.asp ?id=228. Agosto 2014 .

Gandarillas S. H. 2001. Historia de la Investigación para el desarrollo agropecuario en Bolivia: Memorias de un investigador. Ed (s): Gandarillas H., Gandarillas C. y Gandarillas A. (Eds.) Cochabamba, Bolivia. $212 \mathrm{p}$.

Instituto Nacional de Estadísticas. 2014. Bolivia Características de Población y Vivienda: Censo Nacional de Población y Vivienda 2012. http://www.ine.gob.bo:8081/censo2012/. 31pp. Consulta: Julio 2014.

Instituto Nacional de Estadísticas. 2014. Estadísticas económicas. Bolivia: Rendimiento agrícola, según cultivo. http://www.ine.gob.bo/indice/general.aspx?c odigo=40104. Consulta: Agosto 2014 .

Instituto Nacional de Innovación Agropecuaria y Forestal. 2012. Directorio de 
Oferentes de Semilla Certificada e Importada - La Paz. La Paz, Bolivia. 45 p.

Instituto Nacional de Innovación Agropecuaria y Forestal. 2014. Informe técnico de semillas. Cochabamba, Bolivia. 6

p.

Ochoa C.M. 1990. The potatoes of South America: Bolivia. Cambridge University Press. 226 p.

Rodríguez W. 1954. Colección de ecotipos y especies indígenas de papa. Proyecto A-II.C1, Subproyecto A-II-C-1a. Informe técnico Estación Experimental del Altiplano (Belén) 1953-54. Servicio Agrícola Interamericano. La Paz, Bolivia. 8 p.

Torrez W. y H. Foronda. 2008. Producción de semilla de papa dentro del Sistema Nacional de Certificación de Semillas. Revista de Agricultura, Bolivia 60 (43): 3-9.

Urquidi T. 1984. Universidad y Ecología. Cochabamba, Bolivia. 84 p. 